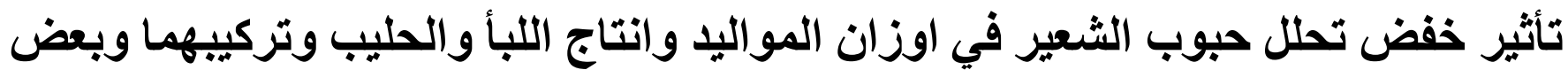

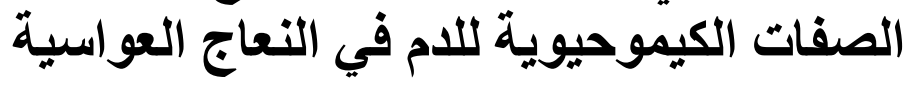

\author{
عمر ضياء الملاح'، عحمد نجم عبدالله ، نادر يوسف عبو 'و سمر احمد توفيق \\ ' قسم الثروة الحيو انية، كلية الزر اعة والغابات، جامعة الموصل، الموصل، ` قسم بحوث الثروة الحيو انية، دائرة البحوث الزر اعية، وزارة \\ الزر اعة، العراق

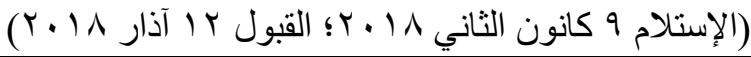

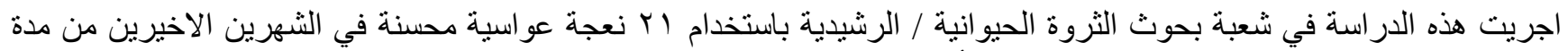

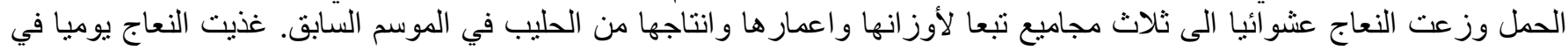

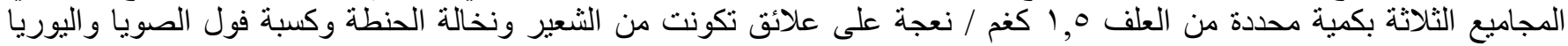

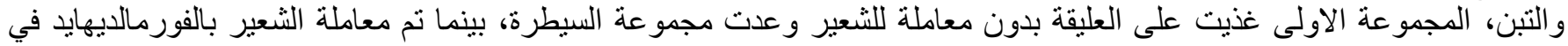

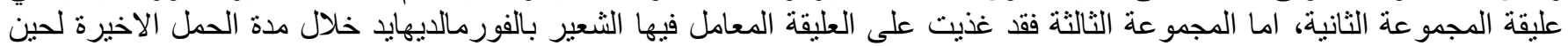

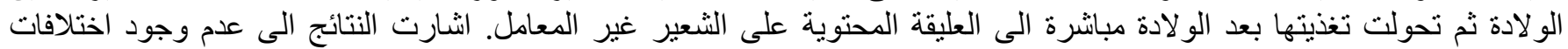

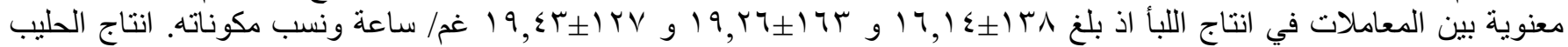

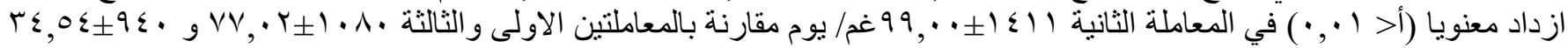

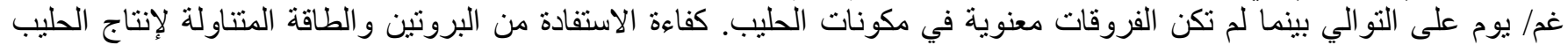

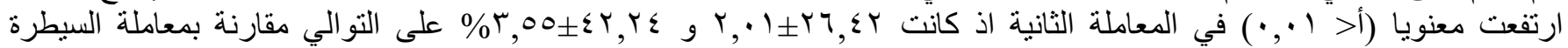

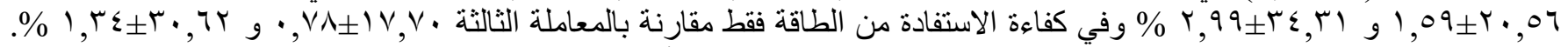

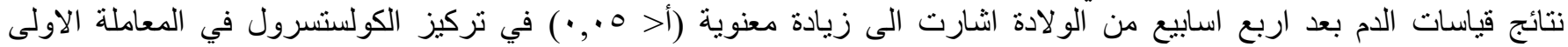

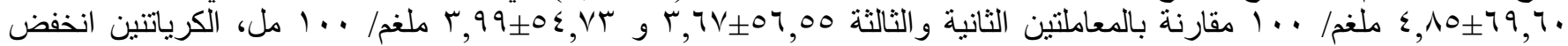

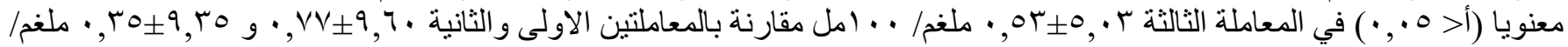

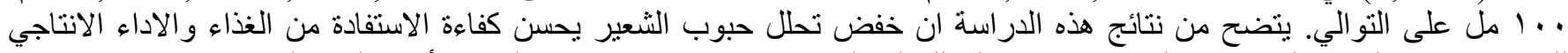

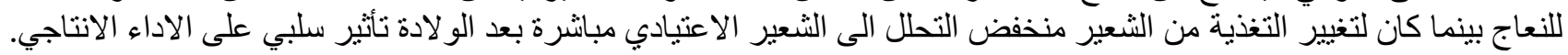

\section{Effect of reducing barley grain degradability on lambs weight, colostrum, milk production, their composition and some biochemical blood trats in Awassi ewes}

\author{
O.D. Almallah ${ }^{1}$, M.N. Abdullah ${ }^{2}$, N.Y. Abbo ${ }^{2}$ and S.A. Tawfeeq ${ }^{2}$ \\ ${ }^{1}$ Animmal Research Department, College of Agriculture and Forestry, University of Mosul, Mosul, ${ }^{2}$ Department of \\ Agriculture Research, Nineveh, State Board of Agriculture Research, Ministry of Agriculture, Iraq
}

\section{Abstract}

This study was conducted in research department of animal resource / Al-Rashidiya, by using 21 of Awassi ewes in the last two months of gestation, ewes were divided according to their body weight, age and milk production during the previous season into three groups, daily feed intake in all groups was restricted with $1.5 \mathrm{~kg}$ / ewe on rations consist of barley, wheat bran, soybean meal, urea and straw. the first group was fed on ration contained barley untreated with formaldehyde (T1), while barley grain treated with formaldehyde in the ration of second group (T2), the third group fed on formaldehyde treated barley 
in late gestation and switch directly after lambing to the untreated barley (T3). Results indicated that differences was nonsignificant in colostrum yield $138 \pm 16.41,163 \pm 19.26$ and $127 \pm 19.43 \mathrm{~g} / \mathrm{hr}$ and in its components, milk yield was increased $(\mathrm{P}<0.05)$ in T2 $1411 \pm 99.04 \mathrm{~g} /$ day as compared with T1 and T2 $1080 \pm 77.02$ and $940 \pm 34.54 \mathrm{~g} /$ day respectively, while no significant differences was noted in milk composition. Efficiency of protein and energy intake for milk production significantly higher $(\mathrm{P}<0.01)$ in $\mathrm{T} 2$ which were $26.42 \pm 2.01$ and $42.24 \pm 3.55 \%$ compared to $\mathrm{T} 1(20.56 \pm 1.59$ and $34.31 \pm 2.99 \%)$ and only in energy efficiency as compared with T3 which were (17.70 \pm 0.78 and $30.62 \pm 1.34 \%)$. Result of blood parameters after four week postpartum was indicated a significant $(\mathrm{P}<0.05)$ increase in cholesterol concentration in $\mathrm{T} 169.60 \pm 4.85 \mathrm{mg} / \mathrm{dl}$ compared to T2 and T3 $56.55 \pm 3.67$ and $54.73 \pm 3.99 \mathrm{mg} / \mathrm{dl}$, blood creatinine concentration decreased $(\mathrm{P}<0.05)$ in $\mathrm{T} 35.03 \pm 0.53$ $\mathrm{mg} / \mathrm{dl}$ as compared to $\mathrm{T} 1$ and $\mathrm{T} 2$ which were $9.60 \pm 0.77$ and $9.35 \pm 0.53 \mathrm{mg} / \mathrm{dl}$ respectively. In conclusion, reducing barley grain degradability improve feed efficiency and ewes performance, while the directly change of the feeding after lambing from low degraded barley to the normal barley grain had a negative effect in performance.

Available online at http://www.vetmedmosul.org/ijvs

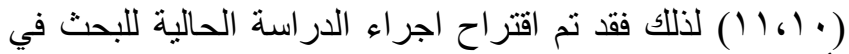
المقدمة

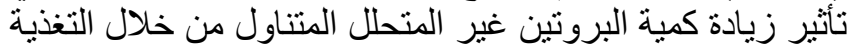

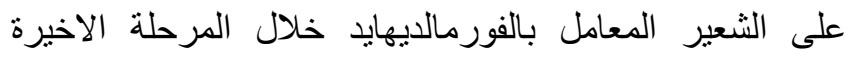

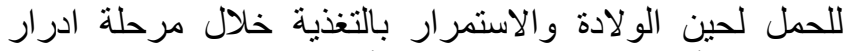

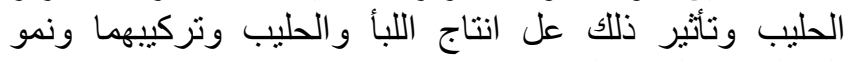
المو اليد في النعاج العو اسية.

\section{المواد وطرائق العمل}

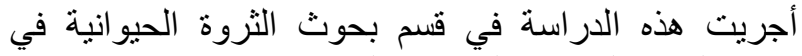

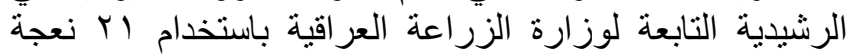

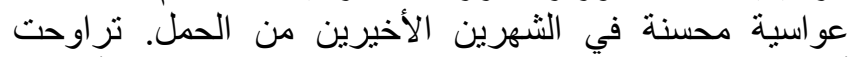

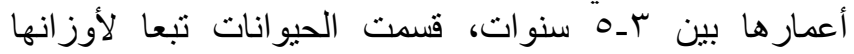

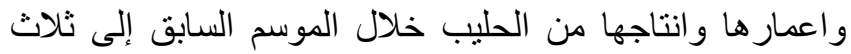

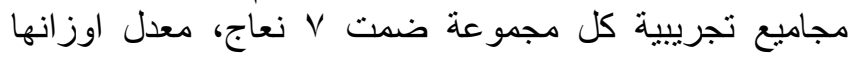

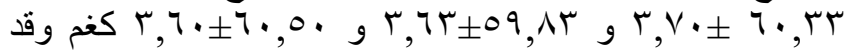
بلغ معدل انتاجها من الحليب في الموسم السابق

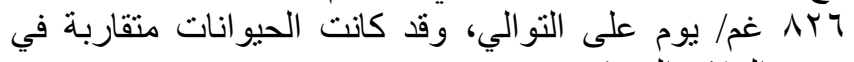

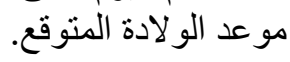

\section{تصميم الاراسة}

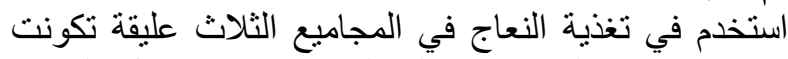

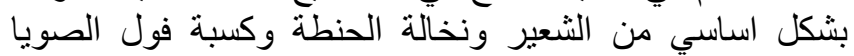

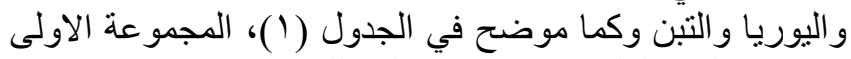

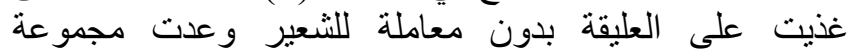

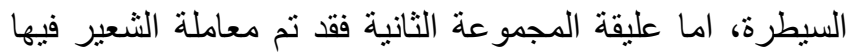

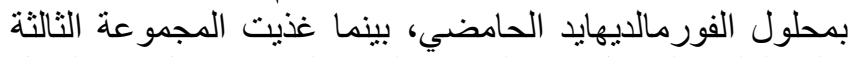

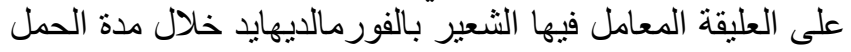

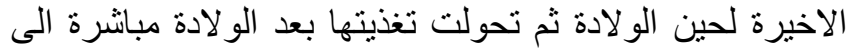

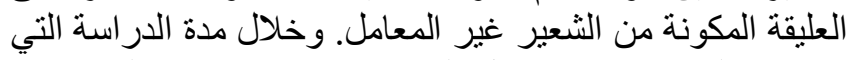

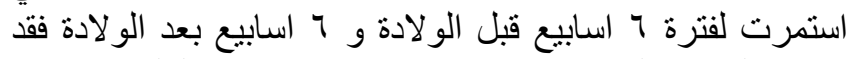

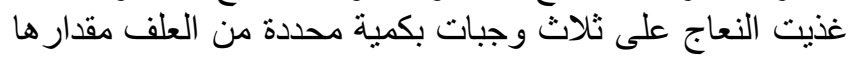

$$
\text { 1,0 }
$$

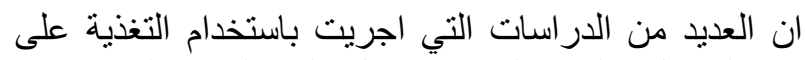

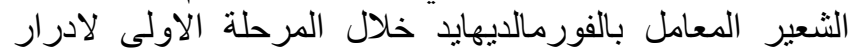

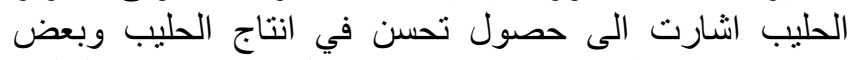

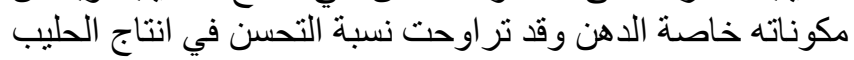

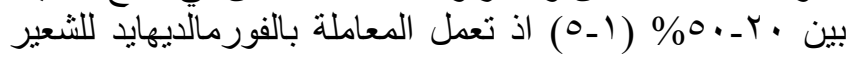

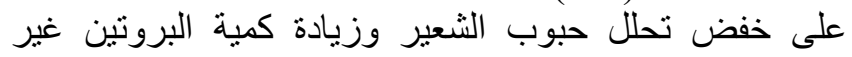

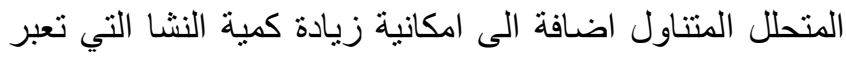

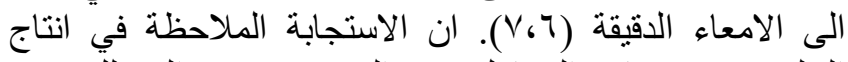

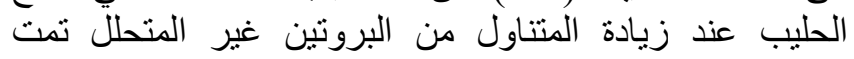

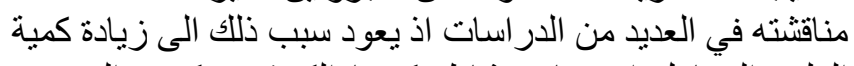

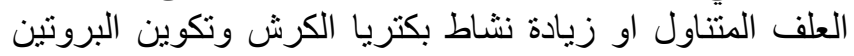

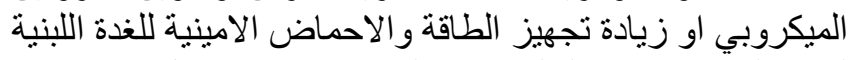

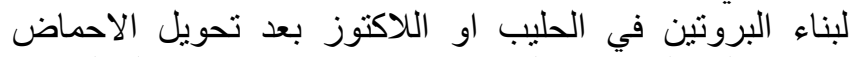

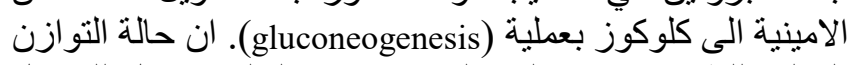

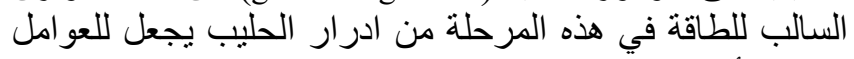

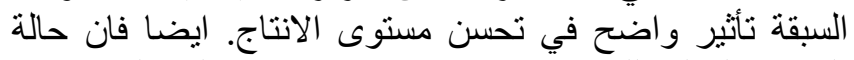

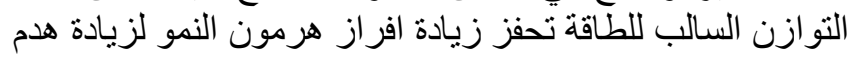

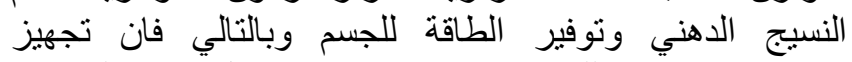

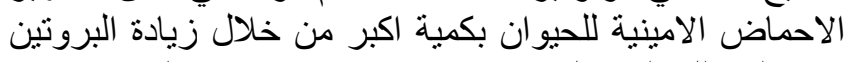

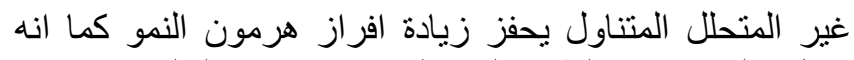

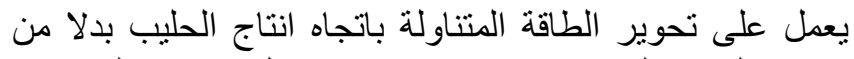

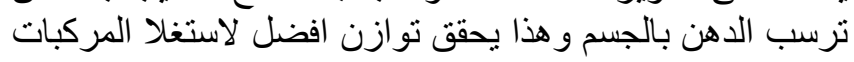

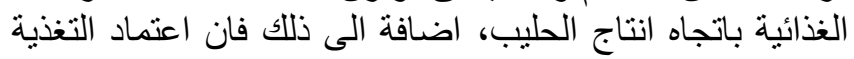

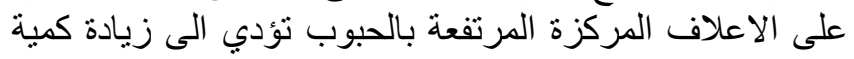

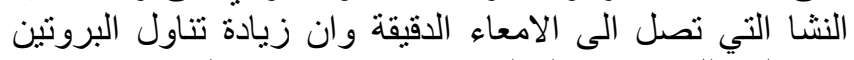

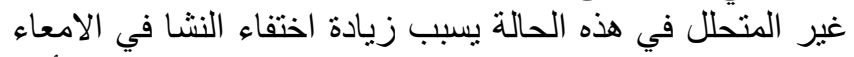

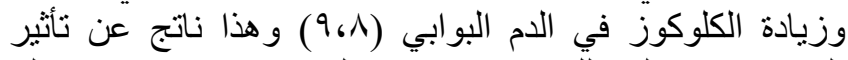

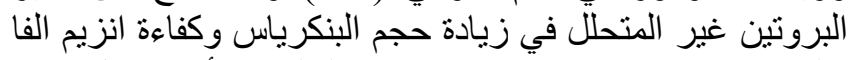

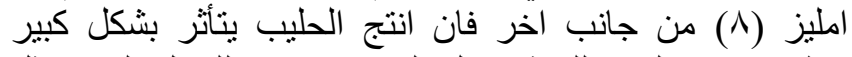

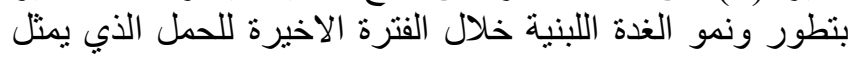
انعكاس لتوازن المركبات الغذائية المتناولة في هذهة اللئنة لألمرحلة 
الجدول (: المكونات والتركيب الكيميائي للعلائق التجريبية

\begin{tabular}{|c|c|c|}
\hline التخفضر & الاعتيادير & المكونات \% \\
\hline --- & 00 & شعبر غير معامل \\
\hline 00 & --- & شعير معامل \\
\hline rq & rq & نخالة حنطة \\
\hline ० & 0 & كسبة فول صويا \\
\hline 9 & 9 & تبن حنطة \\
\hline 1 & 1 & 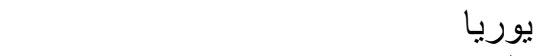 \\
\hline$\cdot, 0$ & $\cdot, 0$ & 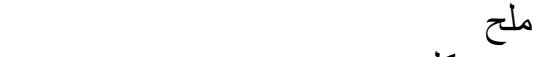 \\
\hline$\cdot, 0$ & $\cdot, 0$ & حجر كلس \\
\hline \multicolumn{3}{|r|}{ التحليل الكيميائي \% } \\
\hline$q \cdot, \cdot v$ & $q \cdot, \cdot V$ & مادة جافة * \\
\hline $9 \leq, 10$ & $9 \leqslant, 10$ & مادة عضوية * \\
\hline$r, \wedge l$ & $r, \wedge l$ & مستخلص الايثر * \\
\hline 17,7 & 17,7 & 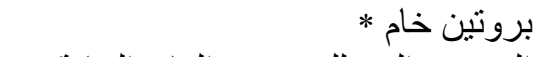 \\
\hline $1 \cdot, \Gamma_{1}$ & $1 r, r$ & البروتين المتحلل \% من المادة الجافة ** \\
\hline $7, r 9$ & $r, o \wedge$ & الجافة*** غير المتحلل \% من المادة \\
\hline $1 \cdot, r$ & $1 \cdot, \cdot r$ & الطاقة الايضية ميكا جول/ كغمْ \\
\hline $1 \cdot, r \wedge$ & 17,99 & بروتين متحلل غم/ ميكاجول \\
\hline $7, r V$ & r,ov & بروتين غير متحلل غم/ ميكاجول \\
\hline \multicolumn{3}{|c|}{ 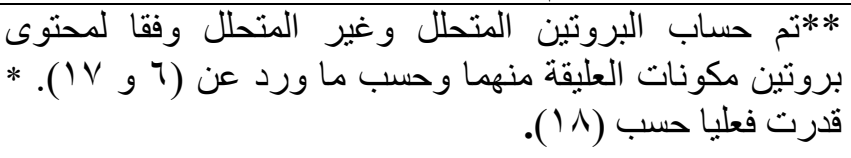 } \\
\hline
\end{tabular}

تم في هذه الدراسة تحديد العلف المتناول في المعاملات

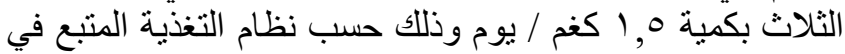

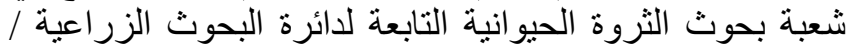

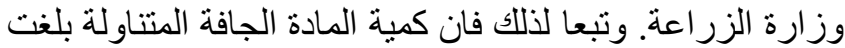

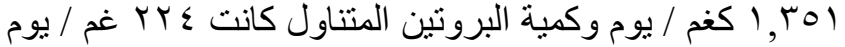

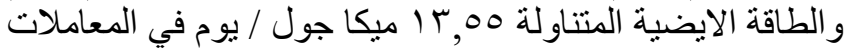

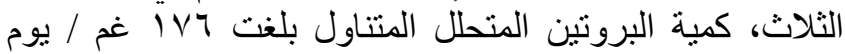

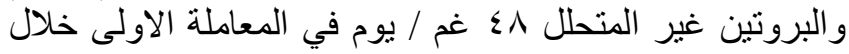

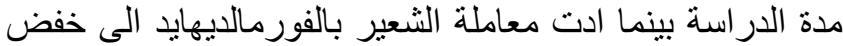

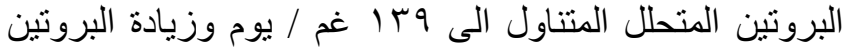

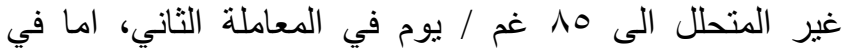

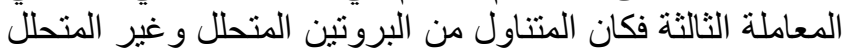

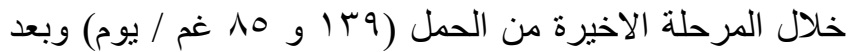

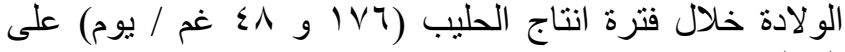

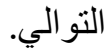

طريقة المعاملة بالفورمالديهايد

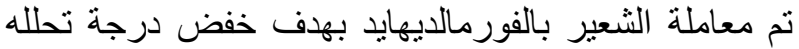

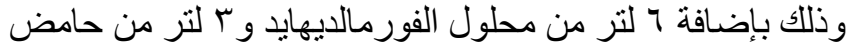

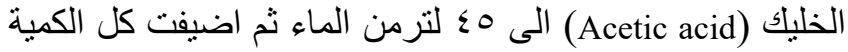

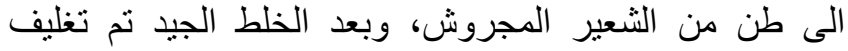

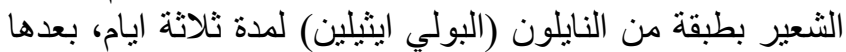

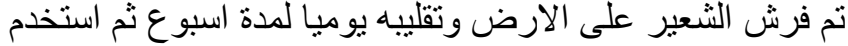

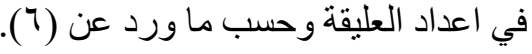

طريقة اخذ القياسات ونماذج التحليل

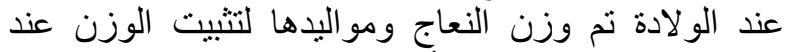

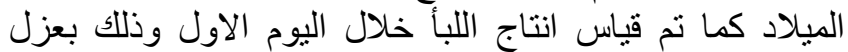

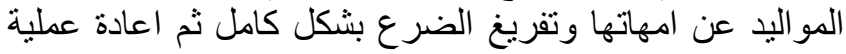

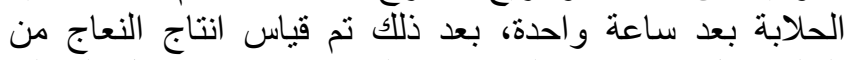

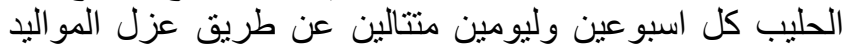

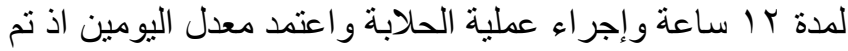

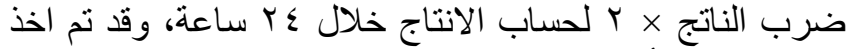

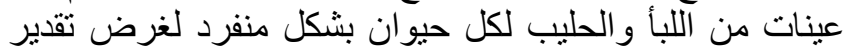

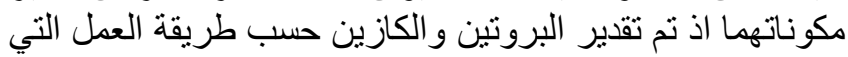

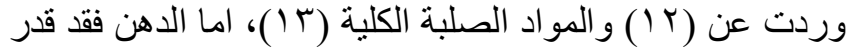

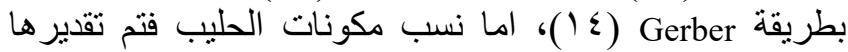

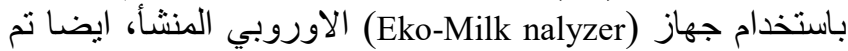

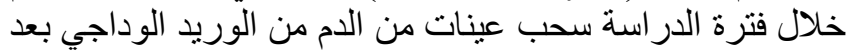

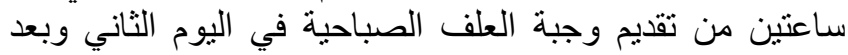

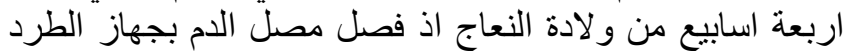

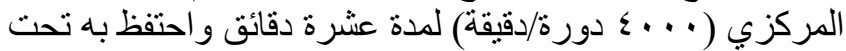

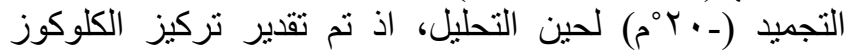

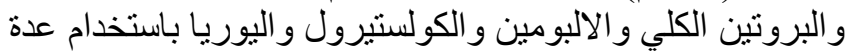
التحليل الجاهزة الفرنسية نوع (Biolabo) بواسطة جهاز قياس بالئ الطيف الضوئي ( Spectrophotometer, Biotech, Engenering,

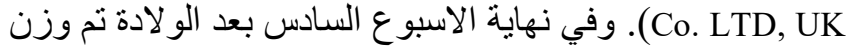

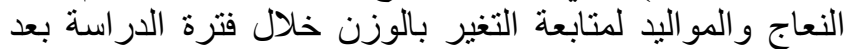

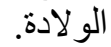

تم إجراء التحليل الإحصائي التصائي للبيانات باستخدام التصميم العشوائي الكامل لتجربة بسيطة باستخدام الحاسوب الإني الإنكتروني

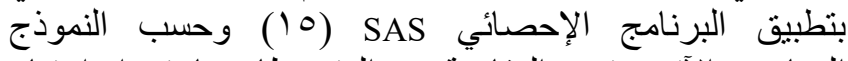
الرياضي الآتي وتمت المقارنة بين المنئ المتوسطات باستخدام اختبار

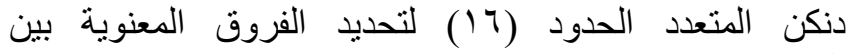

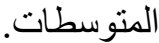

$$
\mathrm{Yij}=\mu+\mathrm{Ti}++ \text { eij }
$$

Yij

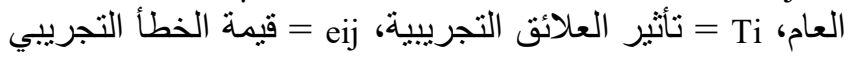
للوحدة التجريبي. 
الكلي انخفض معنويا (أ> ب +,, •) في المعاملة الثالثة اذ بلغ ع

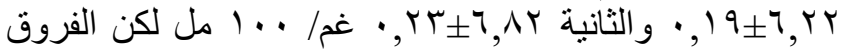

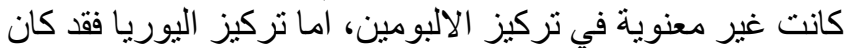

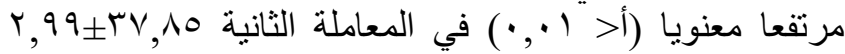

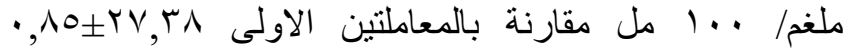

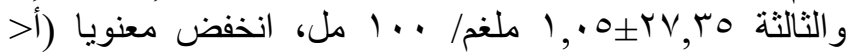

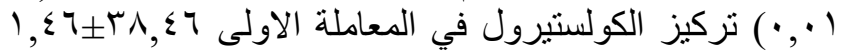

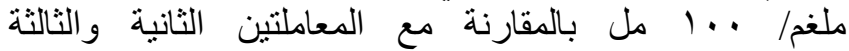

يبين الجدول (r) تأثثر المعاملات التجريبية في انتاج

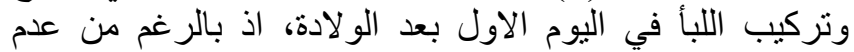

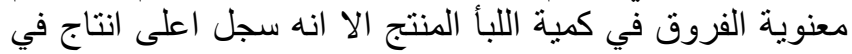

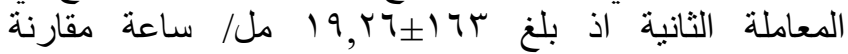

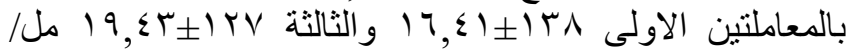
ساعة كذللك كانت الفروقات غير معنى الأنية في نسب البروتين و الكازين و الدهن و المو اد الصلبة الكلية في اللبأ.

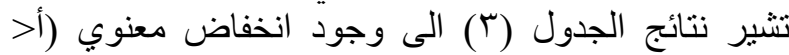

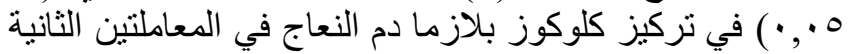
و الثالثة V

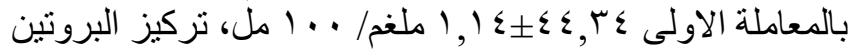

الجدول r : تأثير المعاملات التجريبية في انتاج وتركيب اللبأ خلال ع ساعة من الو لادة

\begin{tabular}{|c|c|c|c|}
\hline المعاملة الثالثة & المعاملة الثانية & المعاملة الاولى & الصفات \\
\hline $19, \varepsilon r \pm 1 r V$ & $19, Y 7 \pm 17 r$ & $17, \xi 1 \pm 1 \pi \wedge$ & انتاج اللبأ غم/ ساعة \\
\hline $1, r r \pm q, 0$ & $\cdot, \varepsilon \Lambda \pm q, r V$ & $\cdot, V \cdot \pm q, V r$ & البروتين \% \\
\hline$\cdot, 97 \pm V, r$. & $\cdot r \Lambda \pm \nu, r_{0}$ & $\cdot, 00 \pm V, V Y$ & الكازين \% \\
\hline$\cdot, r T \pm V, 0$. & $\cdot, 07 \pm V, \cdot V$ & $., O V \pm V, o v$ & الدهن \% \\
\hline$r, \varepsilon v \pm r 0, \cdot \Lambda$ & $1, \wedge r \pm r \wedge, \varepsilon \varepsilon$ & $1,1 \wedge \pm r q, \wedge q$ & المواد الصلبة الكلية \% \\
\hline
\end{tabular}

\begin{tabular}{|c|c|c|c|}
\hline المعاملة الثالثة & بعد الو لادة الثعانية & المعاملة الاولى قياسات الدم في & الصفاتول r: تأثير المعاملات التج \\
\hline 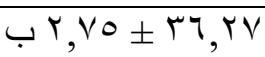 & $\varphi r, r \pm r \varepsilon, \varepsilon V$ & $\mid 1,1 \leqslant \pm \varepsilon \varepsilon, r \leqslant$ & الكلوكوز ملغم/ . . ا مل * \\
\hline$\varphi \cdot r \leq \pm 0, \varepsilon \varepsilon$ & $i \cdot r r \pm \Upsilon, \wedge r$ & $1 \cdot, 19 \pm 7, r r$ & البروتين الكلي غم/ . 1 مل *** \\
\hline$\cdot, \cdot 0 \pm r, V V$ & $\cdot, v \pm r, \wedge 0$ & $\cdot, \cdot \wedge \pm r, \wedge$. & الالبومين غم/ . . 1 مل \\
\hline & $i r, 99 \pm r v, \wedge 0$ & & اليوريا ملغم/ . . 1 مل *** \\
\hline$\{1,01 \pm \leqslant 7, \leqslant 9$ & $I r, T \leqslant \pm 01, r_{0}$ & $\varphi 1, \leqslant \tau \pm r \Lambda, \leqslant \tau$ & الكولستيرول ملغم/ . . 1 مل ** \\
\hline
\end{tabular}

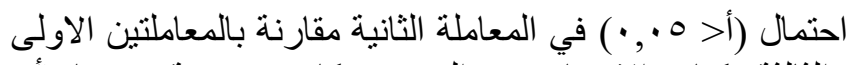

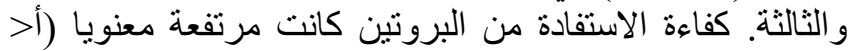

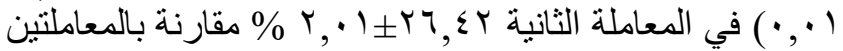

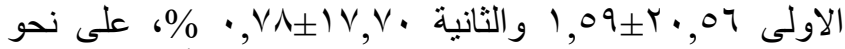

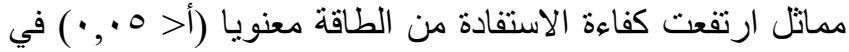

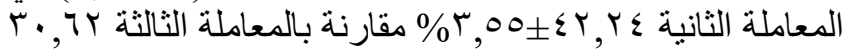
\% \% $9 q_{ \pm} 9$ \%

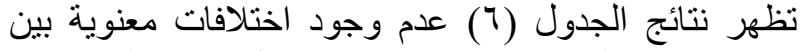

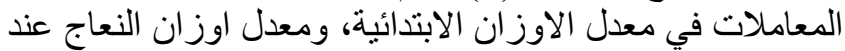

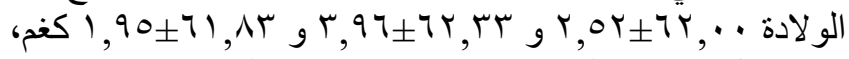

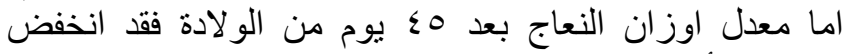

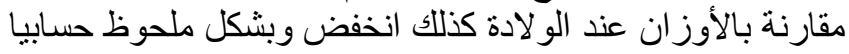

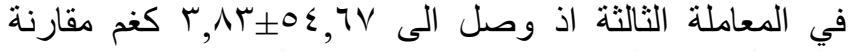

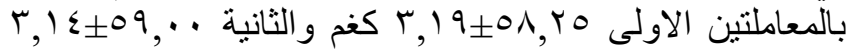

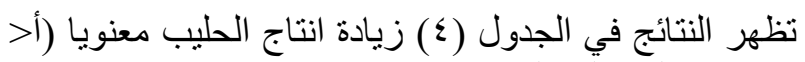

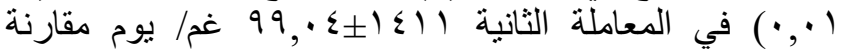

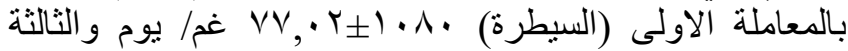
• ع 9 •

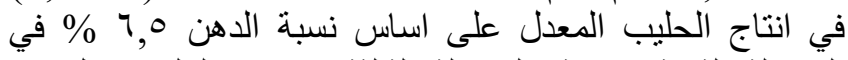

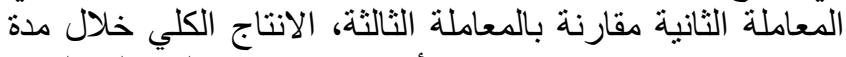

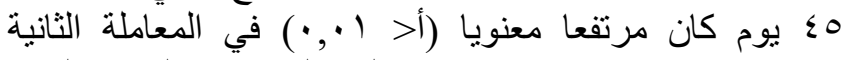

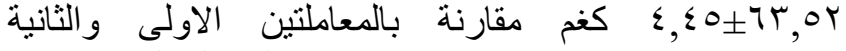
.

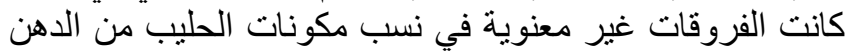
و البروتين والمو اد الصلبة اللادهنية و الطاقة.

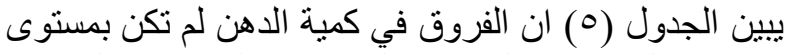

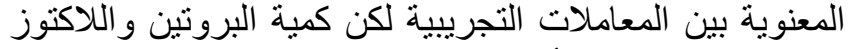

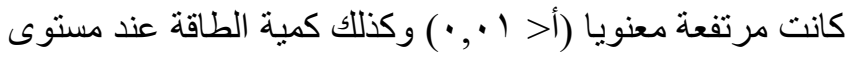


و اليوريا، اما تركيز الكولستيرول فقد انخفض معنويا (آ> 0 •, • )

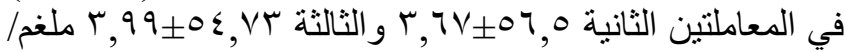

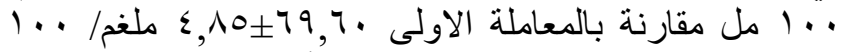

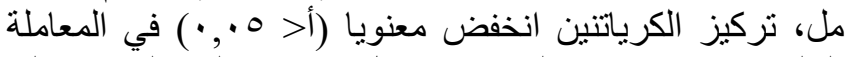

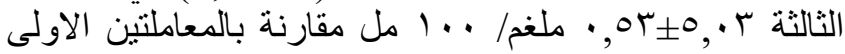

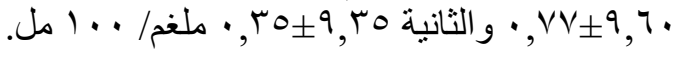

كغم، معدل اوزان المواليد عند الولادة ونهاية مدة التجربة كان

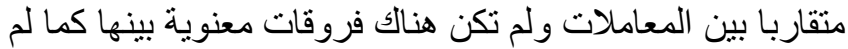

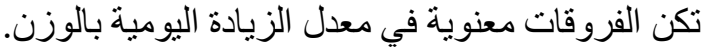

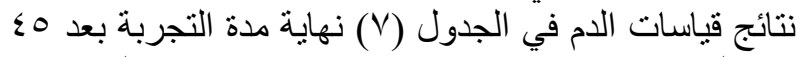

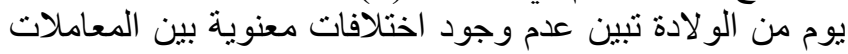

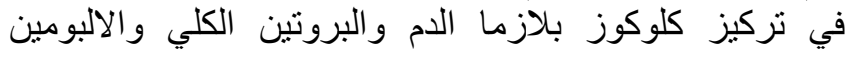

الجدول §: تأثثير المعاملات التجريبية في انتاج الحليب ونسب مكوناته

\begin{tabular}{|c|c|c|c|}
\hline المعاملة الثالثة & المعاملة الثانية & المعاملة الاولى & الصفات \\
\hline$\varphi r \leqslant, 0 \leqslant \pm 9 \leqslant$. & $\mid 99, \cdot \varepsilon \pm 1 \leq 11$ & $\varphi V V, r \pm 1 \cdot \Lambda$. & انتاج الحايب غم/ يوم ** \\
\hline ب & $\{1 \cdot \wedge, 7 \pm 1 r \ldots$ & . . • . & انتاج الحليب المعدل غم/ يوم * \\
\hline ا & $\{\varepsilon, \leqslant 0 \pm 7 r, 0 r$ & $\varphi r, \leqslant\rceil \pm \leqslant \Lambda, \gamma$ & انتاج الحليب الكلي. كغم ** \\
\hline$\cdot, 0 \cdot \pm 7, \vee \vee \leqslant$ & $\cdot r V \pm 0, T V$ & $\cdot, r \pm \pm, r q$ & نسبة الدهن\% \\
\hline$\cdot, \wedge \pm r, \wedge r$ & $\cdot, \cdot \wedge \pm r, \wedge$. & $\cdot, r \pm r, \wedge v$ & نسبة البروتين \% \\
\hline$\cdot, 11 \pm 0,(1$ & $\cdot, T \pm 0, Y \Lambda$ & $\cdot, \cdot 7 \pm 0, V \leqslant$ & نسبة اللاكتوز \% \\
\hline$\cdot r \cdot \pm 1 \cdot, r q$ & $\cdot, r=1 \cdot, \varepsilon \cdot$ & $\cdot, \wedge \pm 1 \cdot, 0$. & نسبة المو اد الصلبة اللادهنية \% \\
\hline$\leq 0, \mid r \pm 1 \cdot v 1$ & $\varepsilon r, \varepsilon \vee \pm 9 \vee \wedge$ & $\varepsilon v, \cdot V \pm 1 \cdot \varepsilon \cdot$ & الطاقة بالحليب كيلو سعرة/ كغم \\
\hline
\end{tabular}

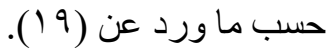

الجدول 0: تأثير المعاملات التجريبية في انتاج الحليب ونسب مكوناته

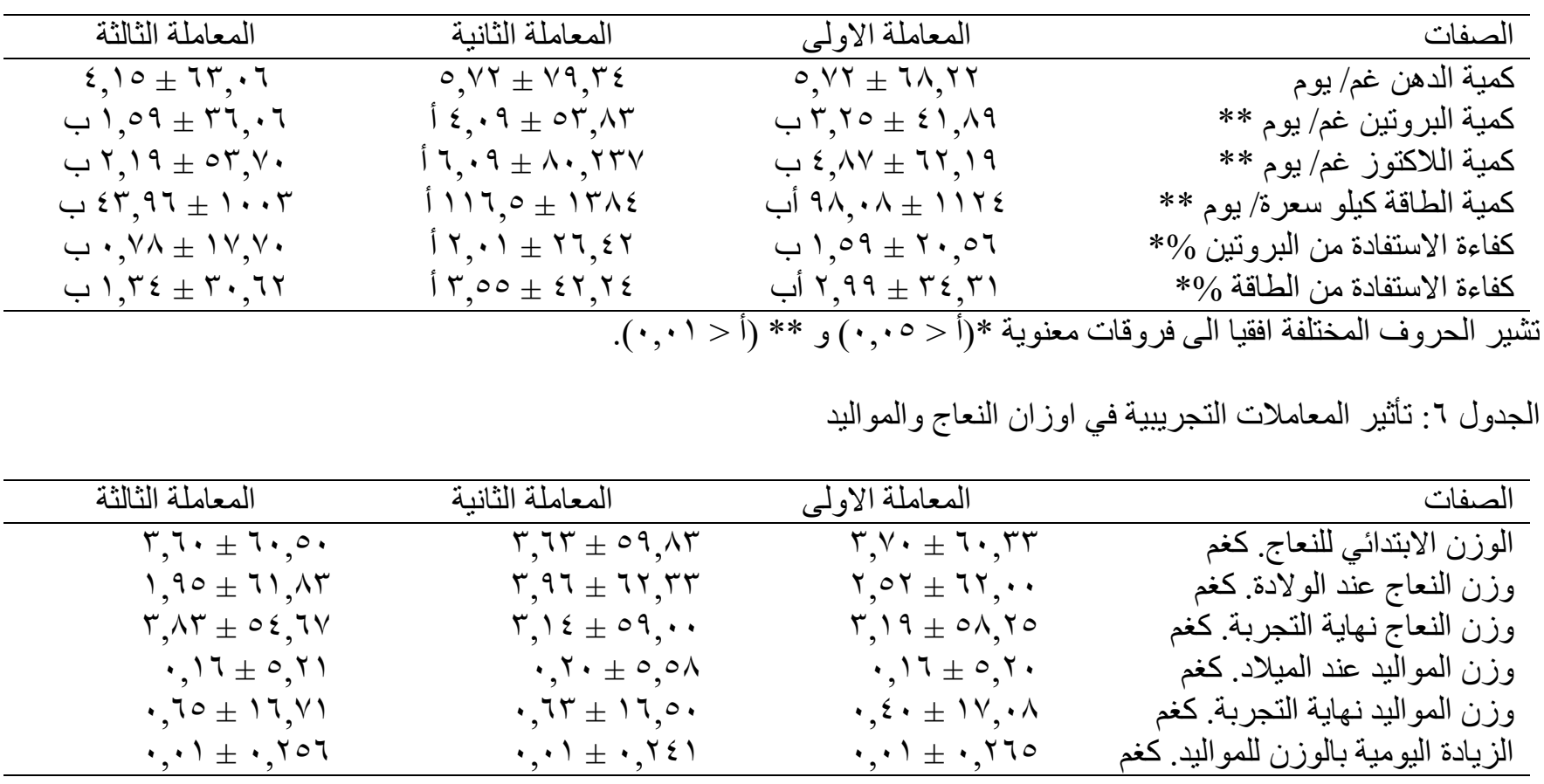


الجدول V: تأثير المعاملات التجريبية في بعض قياسات الدم بعد اربعة اسابيع من الو لادة

\begin{tabular}{|c|c|c|c|}
\hline المعاملة الثالثة & المعاملة الثانية & المعاملة الاولى & الصفات \\
\hline$r, r q \pm \varepsilon \cdot r \varepsilon$ & $1, r \cdot \pm \varepsilon 1,9 V$ & $1, M \pm \leqslant 0, r V$ & الكلوكوز ملغم/ . . 1 مل \\
\hline$\cdot r \wedge \pm \tau, \wedge r$ & $\cdot, r v \pm v, \varepsilon \cdot$ & $\cdot, \leqslant 0 \pm 7, \vee 0$ & البروتين الكلي غم/ . مل \\
\hline$\cdot, r \varepsilon \pm r, 9 q$ & $\cdot r v \pm r, \varepsilon \cdot$ & $\cdot, \leqslant r \pm r, \leqslant r$ & الالبومين غم/ . . ا مل \\
\hline$\varepsilon, r) \pm r q, \varepsilon V$ & $r, \wedge r \pm r \cdot, r \wedge$ & $r, \wedge 0 \pm r \cdot, \varepsilon \cdot$ & اليوريا ملغم/ . 1 مل \\
\hline & 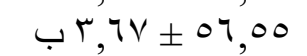 & $\{\varepsilon, 10 \pm 79,7$. & الكولستيرول ملغم/ . . 1 مل * \\
\hline 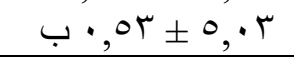 & i. & $i^{\prime} \cdot, V \vee \pm q, 7$. & تركيز الكرياتتين ملغم/ . . 1 مل \\
\hline
\end{tabular}

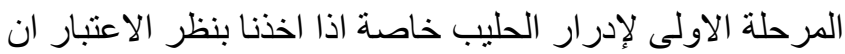

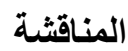

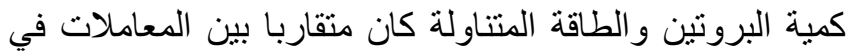

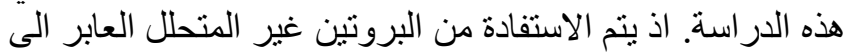

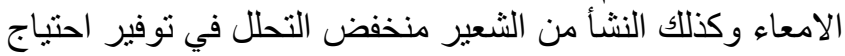

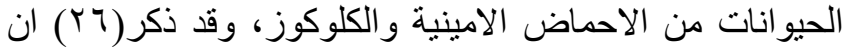

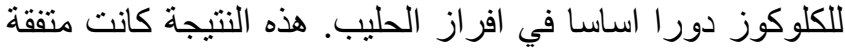

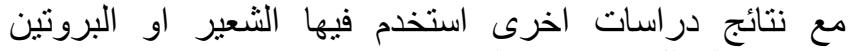

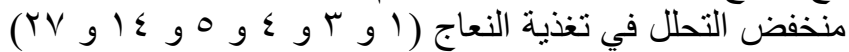

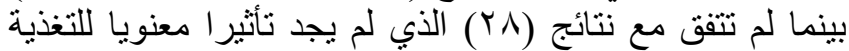

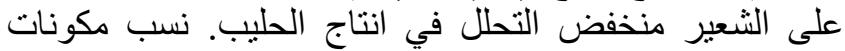
الحليب من البرونين و الدهن والمواد الطئل الصلبة اللادهنية و اللاكتوز

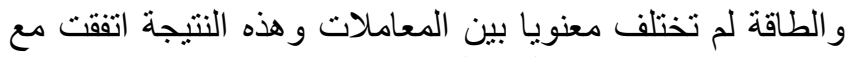

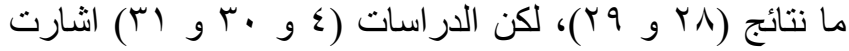

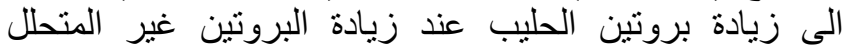

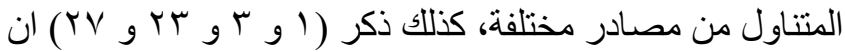

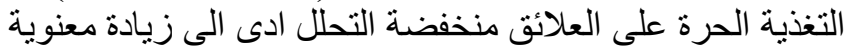

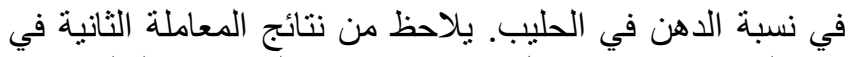

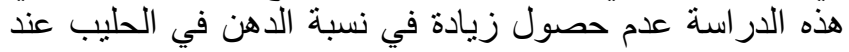

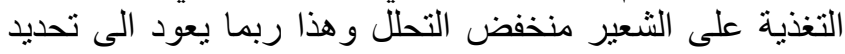

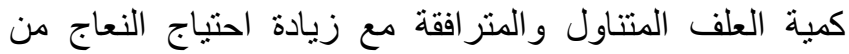

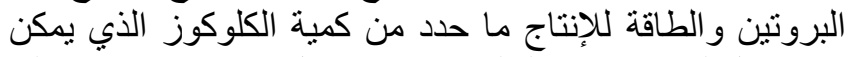

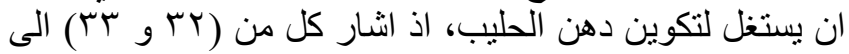

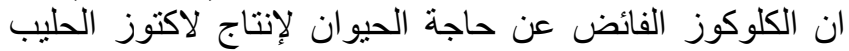

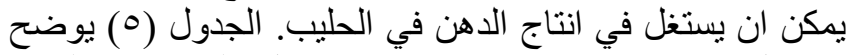

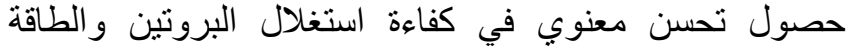

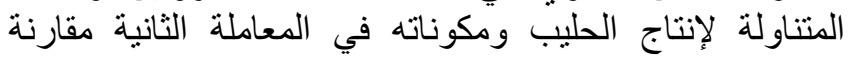

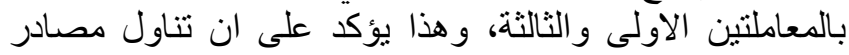

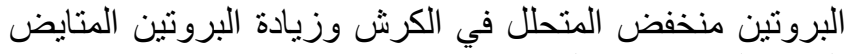

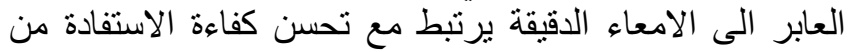

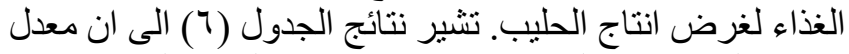

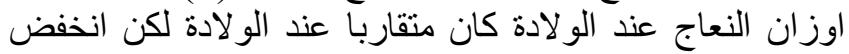

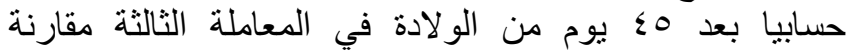

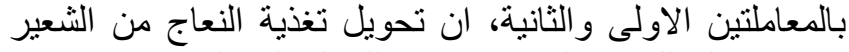
منخفض التحلل في الفترة الاخيرة للحمل الى الثعير الاعتيادي التيدي

تشير نتائج الجدول (Y) الى حصول تحسن وان كان غير النّات

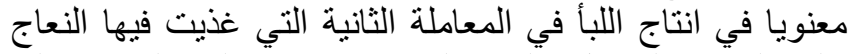

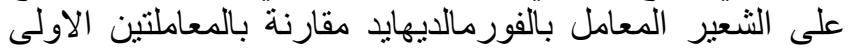

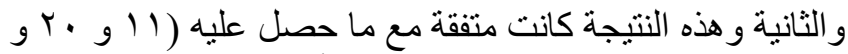

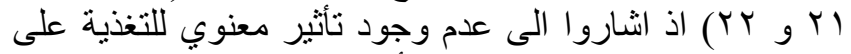

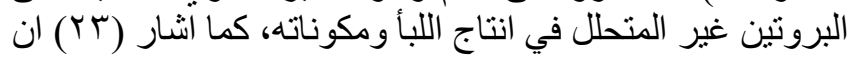

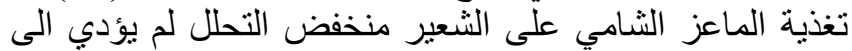

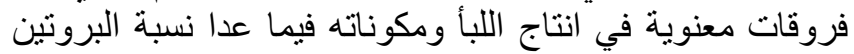

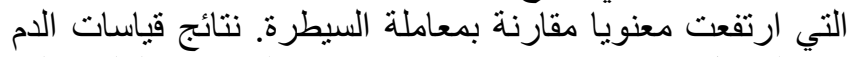

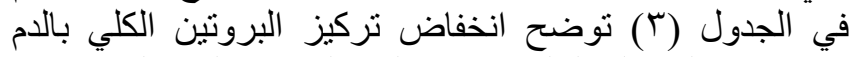

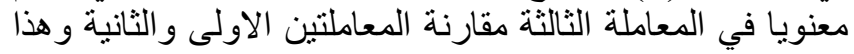

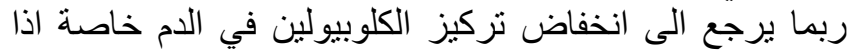

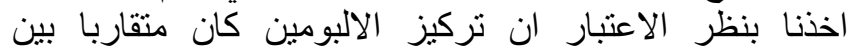

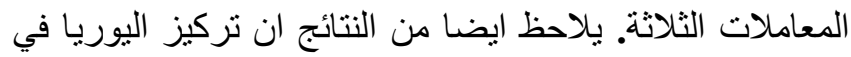

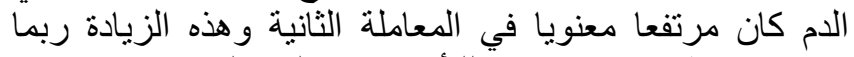

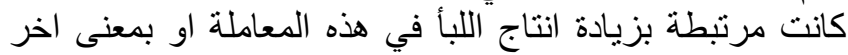

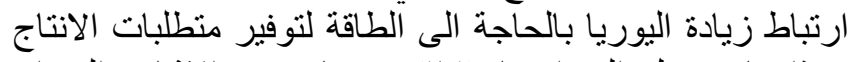

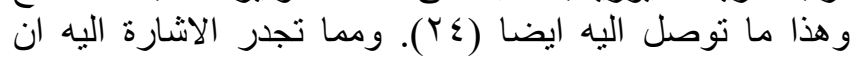

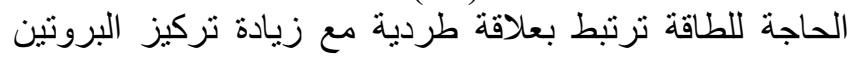

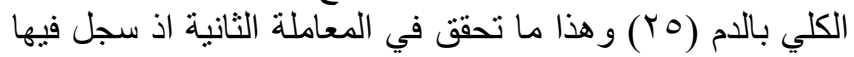

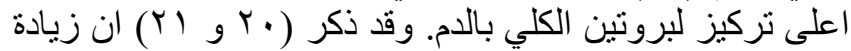

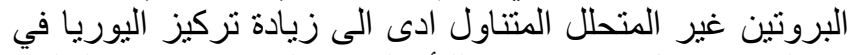

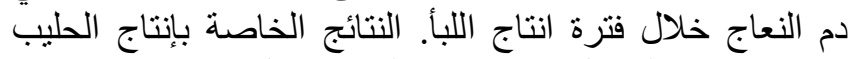

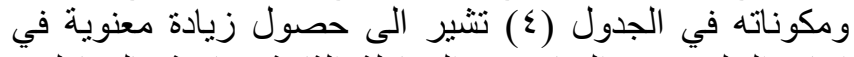

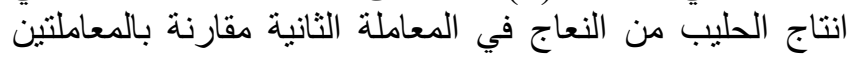

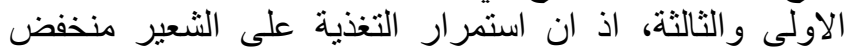

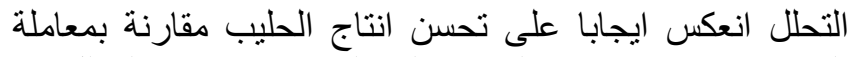

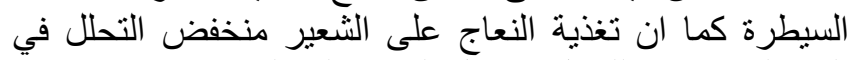

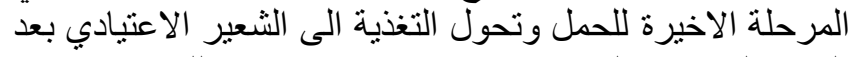

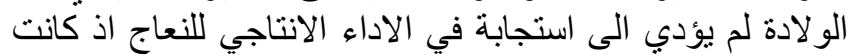

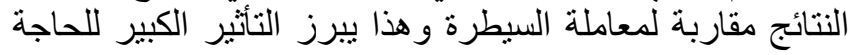

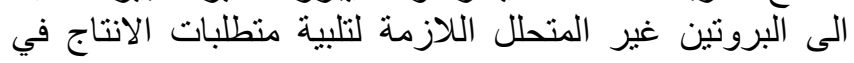


•. المولى، حسين أحمد سليمان عبدالله. ثأثثير التغذية بالعلف المعامل

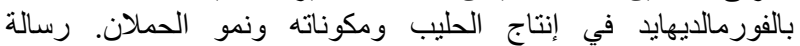

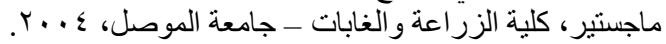

6. Kassem MM, Thomas PC, Chamberlain DG, Robertson S. Silage intake and milk production in cows given barley supplements of reduced ruminal degradability. Grass Forage Sci.1987;42:175-183.

7. Philips GJ, Citron TL, Sage JS, Cummins KA, Cecava MJ, McNamara JP. Adaptation in body muscle and fat in transition dairy cattle fed differing amounts of protein and methionine hydroxyl analog. J Dairy Sci. 2003;86:3634-3647.

8. Richards CJ, Swanson KC, Paton SJ, Harmon DL, Huntington GB. Pancreatic exocrine secretion in steers infused post ruminally with casein and cornstarch. J Anim Sci. 2003;8:1051-1056.

9. Taniguchi K, Huntington GB, Glenn BP. Net nutrient flux by visceral tissues of beef steers given abomasal and ruminal infusions of casein and starch. J Anim Sci. 1995;73:236-249.

10. Bell AW. Regulation of organic nutrient metabolism during transition from late pregnancy to early lactation. J Dairy Sci. 1995;73:28042819.

11. Dawson LE, Carson AF, Kilpatrick DJ. The effect of the digestible undegradable protein concentration of concentrates and protein source offered to ewes in late pregnancy on colostrum's production and lamb performance. Anim Feed Sci Techno. 1990;82:21-36.

12. Foley YJ, Buckley J, Murphy MF. Commercial testing and product control in the dairy industry. University College, Cork. 1974.

13. O'Connor CB. Rural Dairy Technology. ILCA Training manual. International Livestock Research Institute, Addis Ababa, Ethiopia. 1994:133pp.

14. Trout GM, Locus PC. Comparison of Babcock, Gerber, Minnesota, Pennsylvania, and Mojonnier methods for determining the percentage of fat in homogenized milk. Michigan Agric Exp Station. 1946:145159 .

15. SAS. Statistical Analysis System. SAS institute, Inc. Cary. N. C. 2000.

16. Duncan CB. Multiple rang and multiple “ $F$ ” test. Biometric. $1955 ; 11: 1-12$.

17. Stanton TL, LeValley S. Feed omposition For Dairy cattle and sheep. Colorado State University Extension, Livestock Series Management, Fact Sheet No.(1). 2010:615.

18. AOAC. Official Method of Analysis. $17^{\text {th }}$ Ed.(Association of Official Analytic Chemists), Washington, DC, 2002.

19. Pulina G, Bencini R. Dairy Sheep Nutrition. CBAI International Publishing. Biddles Ltd, King's Lynn. UK. 2004.

20. Amanlou H, Karimi, A, Mahjoubi E, and Milis C. Effects of supplementation with digestible undegradable protein in late pregnancy on ewe colostrum production and lamb output to weaning. J Anim Physi Anim Nutr (Berlin). 2011;95:616-622.

21. Banchero GE, Quintans G, Martin GB, Lindsay DR, Milton TB. Nutrition and colostrum production in sheep. 1. Metabolic and hormonal response to high-energy supplement in the final stage of pregnancy. Reprod Fert Develo. 2004;16:1-11.

22. Rezai F, Zamani F, Vatankhah M. Effect of rumen undegradable protein on colostrums quality and growth of Lori Bakhtiari lambs. Global Veter. 2012;8:93-100.

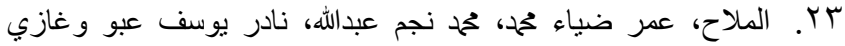

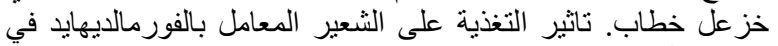

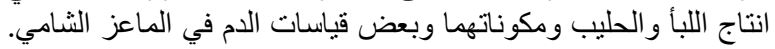

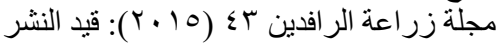

24. El-Sherif MM, Assad F. Changes in some blood constituents of Barki ewes during pregnancy and lactation under semiarid conditions. Small Rum. Res. 2001;40:359-730.

25. Bremmer DR, Bertics SJ, Besong SA, Grummer RR. Changes in hepatic microsomal triglyceride transfer protein and triglyceride in periparturient dairy cattle. J Dairy Sci. 2000;83:2252-260.
بعد الو لادة مباثرة او بمعنى اخر التحديد المفاجئ لكمية البروتين

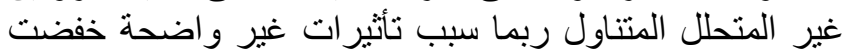

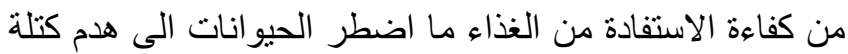

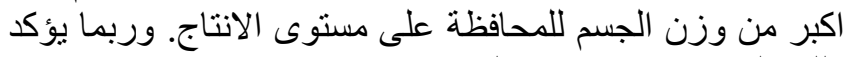

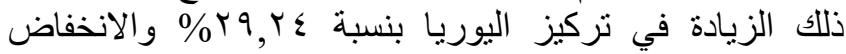

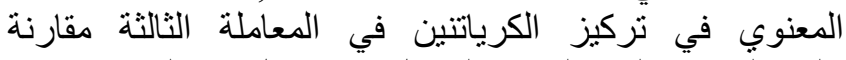

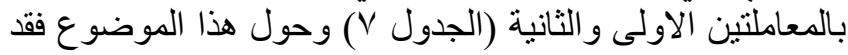

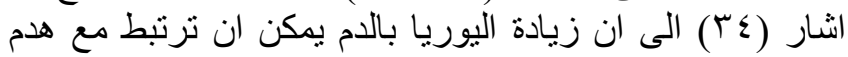

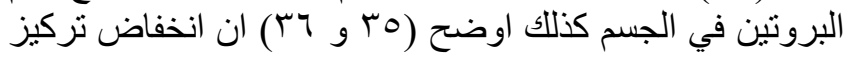

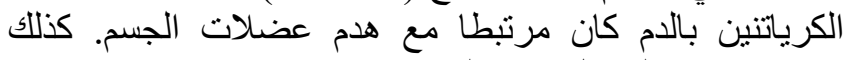

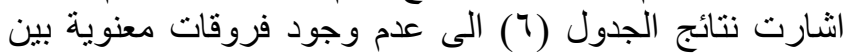

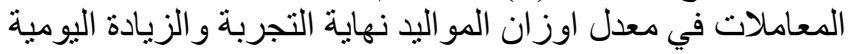

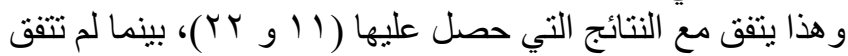

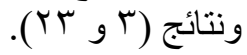

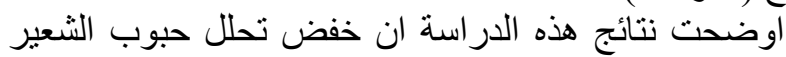

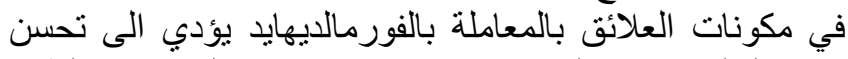

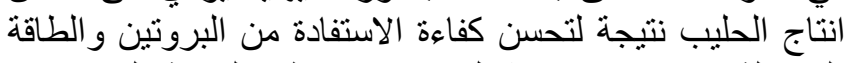

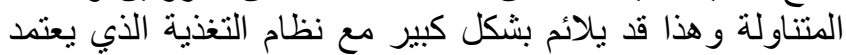

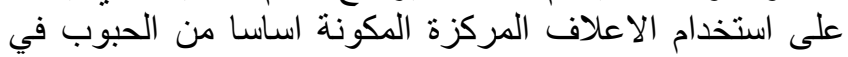

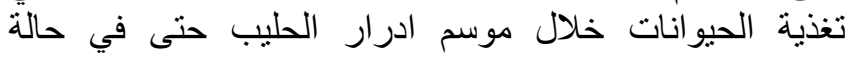

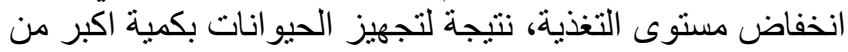

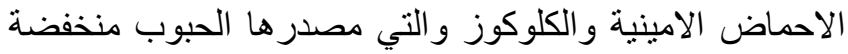

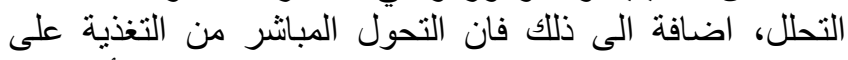

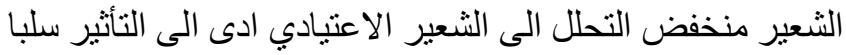

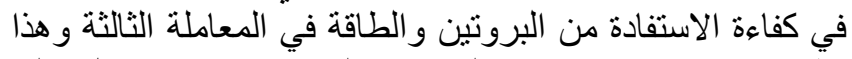

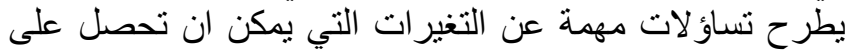

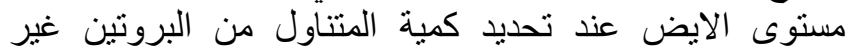

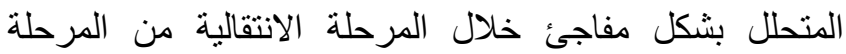

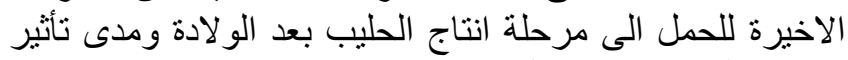
مستوى التغذية في هذه التغير ات.

المصادر

ا ـ الدباغ، رائد حسام عبدالكريم. تاثير اضافة اليوريا اللى العلائق المعاملة

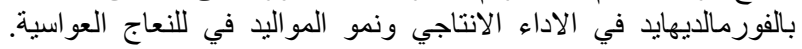

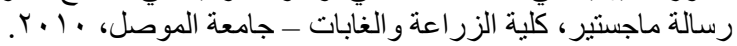

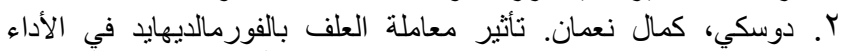

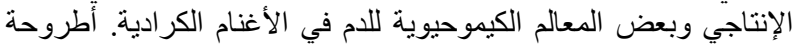

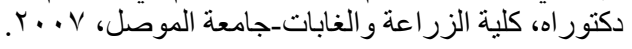

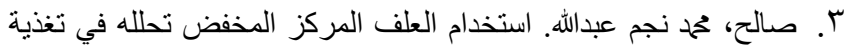

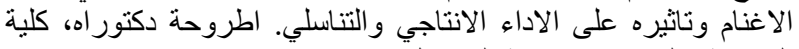

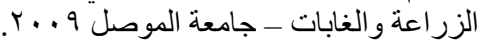

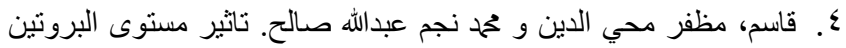

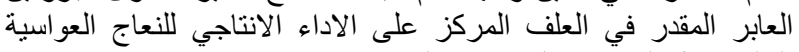

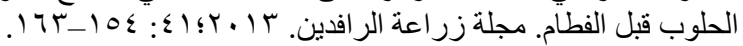


32. Mahr-un-Nisa A, Javaid M, Aasif S, Sarwar M. Influence of varying ruminally degradable to undegradable protein ratio on nutrient intake, milk yield, nitrogen balance, conception rate and days open in early lactating Nili-Ravi buffaloes (Bubalus bubalis). Asian-Aust J Anim Sci. 2008;21:1303-1311.

33. Guinard- Flament J, Delamaire E, Lemosquent S, Boutin M, David Y. Changes in mammary uptake and metabolic fate of glucose with oncedaily milking and feed restriction in dairy cows. Reprod Nutr Develop. 2006;5:589-598.

34. Orskov ER. Energy Nutrition in ruminant. 1991:84-101. (Elsevier, NY).

35. Silanikove N. Effects of heat stress on the welfare of extensively managed domestic ruminants. Livest Prod Sci. 2000;67:1-18.

36. Piccione G, Caola G, Giannetto C, Grasso F, Calanni Runzo S, Zumbo A, Pennisi P. Selected biochemical serum parameters in ewes during pregnancy, post parturition lactation and dry period. Anim Sci Paper Report. 2009;27:321-330.
26. Amanlou H, Karimi, A, Mahjoubi E, and Milis C. Effects of supplementation with digestible undegradable protein in late pregnancy on ewe colostrum's production and lamb output to weaning. J Anim Physi Anim Nutr (Berlin). 2011;95:616-622.

27. Kállai I, Kralovánszky UP. Biology of Meat and Milk Production (in Hungarian). Mezgazdasági Kiadó, Budapest 1975: 201-207.

^ץ. قاسم، مظفر محي الدين. تاثير استخدام الشعير و النخالة المخفض تحلاهيا

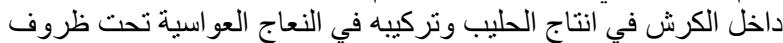

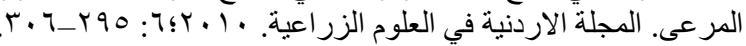

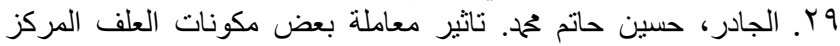

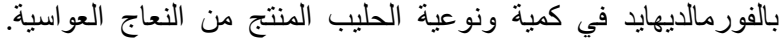

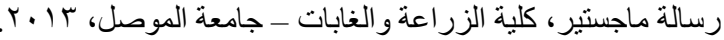

30. Mikolayunas-Sandrock C, Armentano LE, Thomas DL, Berger YM. Effect of protein degradability on milk production of dairy ewes. J Dairy Sci. 2009;92:4507-4513.

31. Aboozar M. Impacts of dietary metabolizable protein on performance and ruminal parameters of Holstein cows at early lactation. Res Opinion Anim Vet Sci. 2012;2:102-108. 